\title{
LETTERS \\ Effect of Perineal Self-Acupressure on Constipation: A Randomized Controlled Trial
}

\author{
Cynthia G. Olsen, MD
}

Boonshoft School of Medicine, Dayton, OH, USA.

J Gen Intern Med 30(4):398

DOI: $10.1007 / \mathrm{s} 11606-015-3195-8$

(c) Society of General Internal Medicine 2015

$\mathrm{T}$ o the Editor: In the recent article by Abbott et. al. (JGIM, Nov 2014), the authors may have misnamed the procedure they employed as "self-acupressure" at the perineal region. If they wish to use this nomenclature then they need to broaden the possible beneficial effects to an acupuncture-style mechanism. ${ }^{1}$ The definition of acupressure is the application of pressure (as with the thumbs or fingertips) to the same discrete points on the body that are stimulated in acupuncture that is used for its therapeutic effects. The locations of these points are usually along energy meridians and they respond similarly to acupressure technique. This is not what the authors describe in the methods section. There is existing literature concerning massage, acupressure, and acupuncture for the treatment of constipation that was not referenced in the article. ${ }^{2}$ The traditional massage technique used in Oriental Medicine, tunia, has been successful for many conditions. ${ }^{3}$ For constipation, this technique is reportedly used on the abdomen, lower back and perineum. The perineal point that the authors describe in their methods section is an important acupoint on the Governor Meridian that runs from the head dorsally to the perineum (GV1), also called Changquiang.
The authors promote the success of their study to peripheral effects such as the manual break-up of hard stools, relaxation of the anal sphincter, and relaxation of the puborectalis, allowing the anorectal angle to straighten and allowing descent of the stool. In acupuncture/acupressure terms, referencing a central nervous system (CNS) effect, there would be a stronger neurobiologic mechanism of action, including an enhanced involuntary coordination of the rectum and sphincter, improved reflexes, reduced anxiety and more self-awareness. If the authors are set in describing their technique as selfacupressure, then they should describe their acupoint correctly (GV1) and consider a possible CNS basis of effect. Otherwise, the technique nomenclature should be changed to self-massage to the perineum. ${ }^{4}$

Corresponding Author: Cynthia G. Olsen, MD; Boonshoft School of Medicine, Dayton, OH, USA (e-mail: cynthia.olsen@wright.edu).

\section{REFERENCES}

1. Abbott R, Ayres I, Hui E, Hui K. Effect of perineal self-acupressure on constipation: a randomized controlled trial. JGIM. 2014. doi:10.1007/ s11606-014-3084.

2. Lamas $\mathbf{K}$, Lindholm $\mathbf{L}$, Stenlund $\mathbf{H}$, et al. Effects of abdominal massage in management of constipation-A randomized controlled trial. Int $J$ Nurs Stud. 2009;46:759-767.

3. Ying $\mathbf{L}, \mathbf{Y u} \mathbf{H}, \mathbf{N i n g} \mathbf{Z}$. Therapeutic observation of warm needling plus tunia for senile functional constipation. J Acupunct Tunia Sci. 2014;12(5):295-299.

4. Dhond RP, Kettner N, Vapanow V. Do the neural correlates of acupuncture and placebo effects differ? Pain. 2008;128(1-2):8-12. 